\title{
Vortex free energy and string Tension at strong and intermediate Coupling
}

Münster, Gernot

First published in:

Physics Letters, Vol. 95B, No. 1, S. 59 - 62, Amsterdam 1980

Münstersches Informations- und Archivsystem multimedialer Inhalte (MIAMI) URN: urn:nbn:de:hbz:6-88419348766 


\section{VORTEX FREE ENERGY AND STRING TENSION AT STRONG AND INTERMEDIATE COUPLING}

Gernot MÜNSTER ${ }^{1}$

II. Institut für Theoretische Physik der Universität Hamburg, Germany

Received 31 March 1980

We present results of high temperature expansions up to order $g^{-24}$ for the vortex free energy respectively string tension in pure lattice gauge theories with gauge groups SU(2) and $Z_{2}$ in 3 and 4 dimensions. For SU(2) in 4 dimensions the resuf calculations of Creutz and is in good agreement. An inten tension smoothly interpolates between strong coupling and weak coupling behaviour.

Gauge field theories have been formulated on a lattice $[1,2]$ in order to study the strong coupling region which is inaccessible to ordinary perturbation theory. In the last years many results have been obtained. It has been proven that lattice gauge theories confine static quarks for strong coupling [3]. On the other hand one would like to know if for gauge group $\mathrm{SU}(2)$ exists with persisting confinement property. Due to asymptotic freedom [4] the continuum limit of $\mathrm{SU}(2)$ or $\mathrm{SU}(3)$ lattice gauge theories is supposed to be related to the weak couplin behaviour $[5,6]$. It is expected to be a limit, where the lattice spacing $a$ and at the same time the bare coupling $g$ go to zero in a way determined by renormalization group equations. The problem of quark confinement in lattice gauge theories is to show that the confining phase extends down to zero coupling.

The connection between strong and weak coupling regions was studied by several authors [7-11]. Of special interest is the intermediate coupling region where a transition from strong coupling to weak coupling behaviour of e.g. the string tension or renormalization group $\beta$-function should take place. Monte Carlo calculations for SU(2) in 4 dimensions [8] indicate a rather sharp changeover. In Mack's theory of quark confinement [11], which is based on the assumption hat confinement of static quarks is due to condensation of vortices [12-14], the transition signals the

${ }^{1}$ Supported by Studienstiftung des Deutschen Volkes. appearance of a nonempty range of distances $a \leqslant d$ $\leqslant d_{\mathrm{c}}(\beta)$ where perturbation theory applies. For values of $\beta$ above it, vortices need a certain thickness $d_{\mathrm{c}}(\beta)$ $>a$ in order to condense. The center $Z_{2}$, respectively $Z_{3}$ of the gauge group plays an important role.

In this letter we discuss the transition between strong and weak coupling with the help of high temperature expansions. We consider euclidean lattice perature expansions. We consider euclidean a $Z_{2}[15]$ gauge theories with gauge groups $S U(2)$ and $Z_{2}[15]$
in 3 and 4 dimensions. The gauge field $U(b) \in S U(2)$ respectively $\sigma(b)= \pm 1$ is attached to links $b$ of a hypercubical lattice with lattice spacing $a$. The action is

$L(U)=\frac{\beta}{2} \sum_{p} \operatorname{tr} U(\dot{p}), \quad$ for $S U(2)$,

$L(\sigma)=\beta \sum_{p} \sigma(\dot{p})$,

for $Z_{2}$,

where $U(\dot{p})$, respectively $\sigma(\dot{p})$ is the product of the group elements on the boundary of an elementary plaquette $p$. The parameter $\beta$ is related to the coupling constant $g$ by

$\beta=4 / g^{2}$.

The sum in eqs. (1) is over all unoriented plaquettes $p$ of the lattice. Expectation values are formed with the path integral measure

$\mathrm{d} \mu=Z^{-1} \exp (L(U)) \prod_{b} \mathrm{~d} U(b)$,

respectively 


$$
\mathrm{d} \mu=Z^{-1} \exp (L(\sigma)) \prod_{b} \mathrm{~d} \sigma(b),
$$

is defined by

Where $Z$ is the partition function and $\mathrm{d} U(b), \mathrm{d} \sigma(b)$ are the Haar measures on the gauge groups.

High temperature i.e. small $\beta$ expansions are obtained by the well-known method of cluster expansion $[16,3]$. It is convenient to expand in terms of the coefficients $c_{j}$ of the series

$$
\exp \frac{1}{2} \beta \operatorname{tr} U=N\left[1+\sum_{j \neq 0}(2 j+1) c_{j}(\beta) \chi_{j}(U)\right]
$$

$\equiv N(1+h(U))$,

$$
\exp \beta \sigma=N^{\prime}(1+k \sigma) \equiv N^{\prime}\left[1+h^{\prime}(\sigma)\right],
$$

where $\chi_{i}, j=1 / 2,1,3 / 2, \ldots$ are the irreducible characters of $\mathrm{SU}(2)$ and $N, N^{\prime}$ are normalization factors. Explicitly

$c_{j}(\beta)=I_{2 j+1}(\beta) / I_{1}(\beta)$,

$k=\tanh \beta$,

where $I_{n}$ are the modified Bessel functions. For $\ln Z$ one obtains expansions in terms of clusters. A cluster $C=\left(X_{1}^{n_{1}}, X_{2}^{n_{2}}, \ldots, X_{i}^{n_{i}}\right)$ consists of a connected set of polymers $X_{1}$ with multiplicities $n_{1}$. Polymers are closed surfaces of plaquettes, which are allowed to possess branching lines. The cluster expansion is

$\ln Z=\sum_{C} b(C) \prod_{i} \frac{\left[A\left(X_{i}\right)\right]^{n_{i}}}{n_{i} !}$,

$A\left(X_{i}\right)=\int \prod_{b} \mathrm{~d} U(b) \prod_{p \in X_{i}} h(U(\dot{p})), \quad$ for $\mathrm{SU}(2)$,

where $b(C)$ are certain combinatorical coefficients and $A\left(X_{i}\right)$ are the so-called activities of polymers. Details will be published elsewhere [18].

An interesting quantity to study is the free energy of vortices per unit length, respectively area $[14,17]$. It is defined in the following way. Consider a finite lattice $L$ of sidelengths $a l_{i}$ in $d=3$ or 4 dimensions. With periodic boundary conditions one gets a partition function $Z_{+}$. A quantum of magnetic vortex flux perpendicular to the $x_{1}-x_{2}$ plane can be introduced in $L$ by applying a singular gauge transformation [13,14,17] leading to twisted boundary conditions. The corresponding partition function is denoted $Z_{-}$. The free energy of a vortex per unit length, respectively area $f\left(l_{1}, l_{2}\right)=\lim _{l_{3}, l_{4} \rightarrow \infty}\left(\prod_{i=3}^{d} l_{i}^{-1}\right)\left(\ln Z_{+}-\ln Z_{-}\right)$.

It is a suitable quantity for the characterization of different phases. In the confining phase $f$ should obey an area law $[14$

$f\left(l_{1}, l_{2}\right) \sim \exp \left(-\alpha a^{2} l_{1} l_{2}\right)$.

In the high temperature expansion of $f$ only a special kind of clusters survive. They contain an odd number of polymers which include a surface cutting $L$ like the expected:

$f\left(l_{1}, l_{2}\right)=2 \exp \left(-\alpha a^{2} l_{1} l_{2}\right)$.

We proved that the coefficient $\alpha$ is in the high temperature expansion equal to the string tension [18], i.e. the corresponding coefficient in the area law for the Wilson loop expectation value $[1,3]$. It gives the slope of the linear potential between static quarks. A direct expansion for the string tension can be set up. We calculated the series up to order $\beta^{12}$. The results are, with $u=c_{1 / 2}, v=c_{1}, w=c_{3 / 2}$ : for SU(2) in $d=3$ dimensions $a^{2} \alpha=-\ln u-2 u^{4}+4 u^{6}-6 u^{4} v-10 u^{8}$

$+4 u^{10}+24 u^{8} v-36 u^{6} v^{2}-6 v^{5}-\frac{602}{3} u^{12}$

$+204 u^{10} v-36 u^{8} v^{2}-24 u^{6} v^{3}-24 u^{4} v^{4}$

$+18 v^{6}-8 u^{9} w-12 v^{5} w u^{-1}$,

for $\mathrm{SU}(2)$ in $d=4$ dimensions

$a^{2} \alpha=-\ln u-4 u^{4}+8 u^{6}-12 u^{4} v-56 u^{8}$

$+120 u^{10}-168 u^{8} v-72 u^{6} v^{2}-12 v^{5}-\frac{4732}{3} u^{12}$

$+888 u^{10} v-396 u^{8} v^{2}-48 u^{6} v^{3}-48 u^{4} v^{4}$

$+36 v^{6}-48 u^{9} w-24 v^{5} w u^{-1}$

for $\mathrm{Z}_{2}$ in $d=3$ dimensions

$a^{2} \alpha=-\ln k-2 k^{4}-2 k^{6}-10 k^{8}-16 k^{10}-\frac{242}{3} k^{12}$,

for $\mathbf{Z}_{2}$ in $d=4$ dimension

$a^{2} \alpha=-\ln k-4 k^{4}-4 k^{6}-56 k^{8}-144 k^{10}-\frac{3616}{3} k^{12}$.

The string tension for $\mathrm{SU}(2)$ in $d=3$ dimensions has $x_{1}-x_{2}$ plane. The expansion leads to an area law as been calculated previously [9] up tó order $\beta^{14}$. Our results agree with theirs.

The case of $\mathrm{SU}(2)$ in $d=4$ dimensions is most interesting. It can be compared with the Monte Carlo simulations of Creutz [8]. In fig. 1 we have plotted $a^{2} a$ according to eq. (11) and the points given in ref. [8] The high temperature series is in very good agreement with the Monte Carlo data in the intermediate coupling region between $\beta=1.6$ and $\beta=2.25$. At $\beta=2.25$ the cur $A$ match $\beta=2.3$ the sting tens Creutz. Above $\beta=2.3$ the string tension seems to follow the weak coupling asymptotic freedom behaviour. This cannot be expected to be reproduced by the high temperature series (11), which in fact goes through zero near $\beta=2.4$.

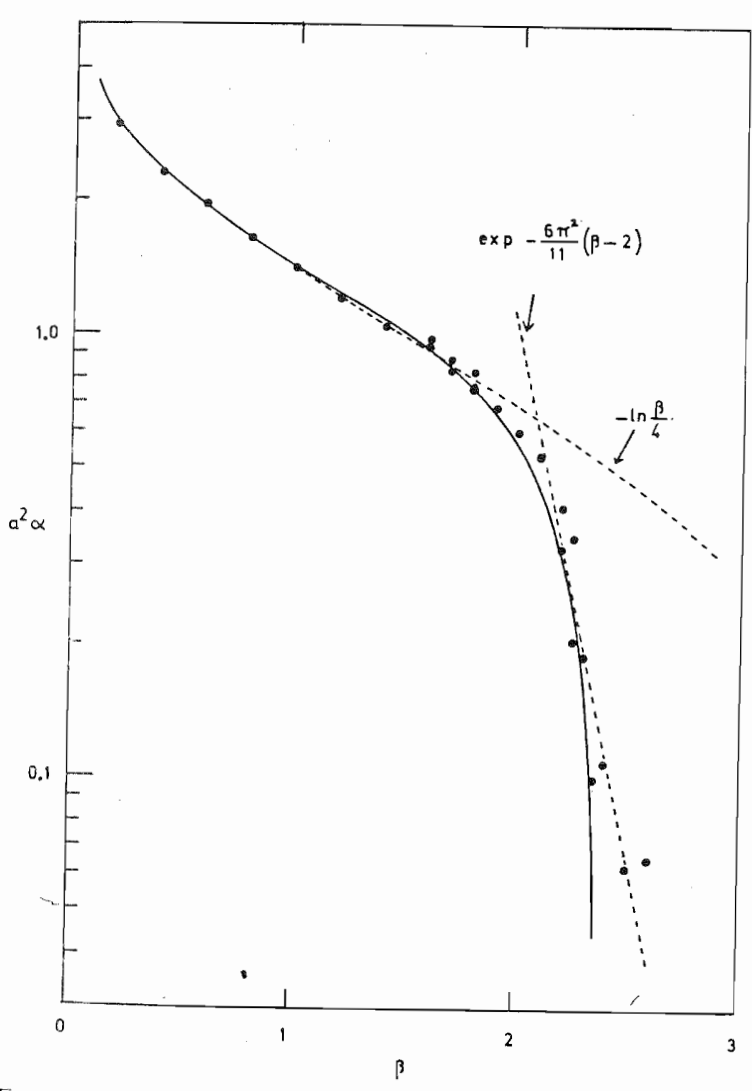

Fig. 1. The string tension $\alpha$ times the lattice spacing square as a function of $\beta=4 / \mathrm{g}^{2}$ for $\mathrm{SU}(2)$ lattice gauge theory in $d$ expansionsions. The solid line represents the result of cluster results of up to 12th order [formula (11)]. The dots are lines are the lonte Carlo calculation of Creutz [8]. The dashed coupling fit of ref. [8].
It should be noted that the cluster expansion series $(10-13)$ for $\alpha$ are not power series in $\beta$. Instead by using the expansion parameters $0 \leqslant c_{j}<1$ one is doing a partial resummation of the usual high temperature series in $\beta$. This appears to improve the convergence properties.

In conclusion we see that high temperature cluster expansions yield accurate values of the string tension at strong and intermediate coupling and their prediction joins smoothly to the expected behaviour at weak coupling.

I would like to thank Prof. G. Mack for many discussions.

Note added. After completion of this work we received a paper by N. Kimura, Critical properties of $\mathrm{Z}(2)$ lattice gauge systems from strong-coupling expansions (Hokkaido University HOU-HP-80-01), where the high temperature series for the string tension of $\mathrm{Z}_{2}$ lattice gauge theory has been calculated up to order $k^{14}$ for $d=3,4$ and 5 . Our results agree with theirs.

References

[1] K. Wilson, Phys. Rev. D10 (1974) 2445.

[2] J. Kogut and L. Susskind, Phys. Rev. D11 (1975) 395. 3) K. Osterwalder and E. Seiler, Ann. Phys. (NY) 110

(1978) 440

[4] G. 't Hooft (1972), unpublished; H. Politzer, Phys. Rev. Lett. 30 (1973) 1346; D. Gross and F. Wilczek, Phys. Rev. Lett. 30 (1973)

[5] J. Kogut, Rev. Mod. Phys. 51 (1979) 659

low the Pauge theory models at publised in: Recont Des G. 't Hooft et 11. (Plenum, New York, 1980).

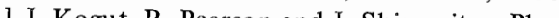

, Phys. Rev. Lett. M. Creutz, Solving

print BNL-26847 (Brookh SU(2) gauge theory, pre-

.

[10] C. Callan, R. Dashen and D. Gross, Phys. Rev. Lett. 44 (1980) 435 .

G. Mack, Predictions of a theory of quark confinement, DESY 80/21 (March 1980).
J. Glimm and A. Jaffe, Nucl. Phys. B149 (1979) 49;

D. Förster, Phys. Lett. 76B (1978) 597.
[12] G. 't Hooft, Nucl. Phys. B1 38 (1978) 1; 
[13] G. Mack and V.B. Petkova, Ann. Phys. (NY) 123 (1979)

442; finement of static quarks by a vortex condensation mechanism, DESY-report 78/69 (Nov. 1978), to be published in Ann. Phys. (NY).

14] G. 't Hooft, Nucl. Phys. B153 (1979) 141

15] F. Wegner, J. Math. Phys. 12 (1971) 2259

R. Balian, J. Drouffe and C. Itzykson, Phys. Rev. D11 (1975) 2098
16] C. Domb and M. Green, eds., Phase transitions and critical phenomena, Vol. 3 (Academic Press, New York, 1974). ] G. Münster, On the characterization of the Higgs phase in lattice gauge theories, DESY-report 79/72 (Nov. 1979). 18] G. Münster, Ph.D. thesis, Universität Hamburg (1980), in preparation. 